\title{
An evidence map of clinical practice guideline recommendations and quality on diabetic retinopathy
}

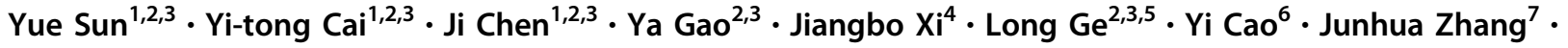 \\ Jinhui Tian $\mathbb{1}^{1,2,3}$
}

Received: 6 December 2019 / Revised: 12 April 2020 / Accepted: 27 May 2020 / Published online: 24 June 2020

(c) The Author(s), under exclusive licence to The Royal College of Ophthalmologists 2020

\begin{abstract}
To present an evidence map for explicating research trends and gaps, we systematically review clinical practice guidelines (CPGs) on diabetic retinopathy (DR) and assess the quality of CPGs and consistency of recommendations. A literature search was performed in PubMed, Embase, Web of Science, CPG databases, and website of diabetes society to include the CPGs. The basic information, methodological quality, and reporting quality of CPGs, recommendations for DR were exacted by the Excel 2013. Methodological and reporting quality of DR CPGs were evaluated by AGREE II instrument and RIGHT checklist. The bubble plot format of evidence map was made by Excel 2013. Nineteen CPGs proved eligible, which included eight DR CPGs and 11 comprehensive diabetic CPGs. The identified CPGs were of mixed quality and they scored poorly in the rigor of development, applicability domains by AGREE II. Field two (background) had the highest reporting rate (86.31\%) and field five (review and quality assurance) obtained the lowest reporting rate (31.58\%) among the seven domains of RIGHT checklist. According to the recommendations of CPGs, there were three inconsistencies in the screening of DR, and CPGs recommendations for treatment were consistent on the whole. At the same time, recommendations for laser therapy were not accurate. Some recommendations were not specific and clear in some DR CPGs. This evidence map could collect and evaluate the characteristics of published CPGs, add to our knowledge and promote the development of trustworthy CPGs for DR.
\end{abstract}

\section{Introduction}

Globally, diabetes is an important public health problem. The World Health Organization has long listed diabetes as one of the leading causes of death worldwide. From 2010 to 2030,

These authors contributed equally: Yue Sun, Yi-tong Cai

Supplementary information The online version of this article (https:// doi.org/10.1038/s41433-020-1010-1) contains supplementary material, which is available to authorized users.

Junhua Zhang

zjhtcm@foxmail.com

$\triangle$ Jinhui Tian

tjh996@163.com

1 Evidence-Based Nursing Center, School of Nursing, Lanzhou University, Lanzhou City, Gansu Province, China

2 Evidence-Based Medicine Center, School of Basic Medical Sciences, Lanzhou University, Lanzhou City, Gansu Province, China there will be a $69 \%$ increase in the number of adults with diabetes in developing countries and a $20 \%$ increase in developed countries [1]. By 2030, the global prevalence of diabetes will exceed 522 million, with one-third of diabetics having diabetic retinopathy (DR) $[2,3]$, and more and more people will suffer from DR $[4,5]$. DR is a complex retinal vascular disease characterized by increased vascular permeability, retinal ischemia and oedema, and neovascularization. In the early stages of the disease, the clinical manifestations of DR are not obvious, usually without any symptoms, but if left untreated, it will eventually lead to blindness [6].

3 WHO Collaborating Center for Guideline Implementation and Knowledge Translation, Lanzhou, China

4 School of Geology Engineering and Geomatics, Chang'an University, Xian City, Shanxi Province, China

5 School of Public Health, Lanzhou University, Lanzhou City, Gansu Province, China

6 The First Affiliated Hospital of Xi'an Jiaotong University, Xian City, Shanxi Province, China

7 Evidence-Based Medicine Center, Tianjin University of Traditional Chinese Medicine, Tianjin, China 
Diabetes is responsible for about 2.5 million cases of blindness worldwide [7]. It has serious implications for the health and quality of life of people around the world [8]. DR can lead to mobility problems and sometimes depression, which can lead directly to poor quality of life [9]. In the United States in 2004, the direct medical cost of blindness caused by DR was about $\$ 500$ million and it is also costeffective to prevent DR [10]. Therefore, the treatment and prevention of DR are becoming more and more significant.

Clinical practice guidelines (CPGs) are statements of system development to help doctors and patients make appropriate health care decisions in specific clinical situations, and the adoption and use depend largely on the development of CPGs $[11,12]$. Well-developed CPGs are the most credible evidence [13]. Nowadays, more and more organizations conduct their own CPGs and publish recommendations. Various studies have shown that CPGs could improve health care and provide recommendations for clinical decision [14, 15]. However, CPGs from different organizations may give conflicting recommendations, which could result in confusion and raise concern about the quality of the CPGs $[16,17]$. Hence, we conducted a systematic evaluation of the authoritative CPGs for DR, including the evaluation and arrangement of the quality and consistency of recommendations.

Evidence mapping is an emerging tool that is increasingly used to quantify and summarize the literature [18]. We use the evidence mapping method to present different recommendations of CPGs for DR, which could better help promote evidence-based decision making [19].

\section{Methods}

\section{Data sources and search strategy}

CPGs were searched in PubMed, Embase, and Web of Science using medical subject headings and keywords. In addition, we searched the CPG databases-Guideline International Network, National Institute for Health and Care Excellence (NICE), Scottish Intercollegiate Guidelines Network (SIGN), and National Guideline Clearinghouse. The official websites for diabetes society were searched to identify additional CPGs which could have been missed.

\section{Eligible criteria and data extraction}

CPGs had to meet the definition of CPGs from the Institute of Medicine [20]. CPGs should be defined as documents developed by a nationally recognized committee, or a medical society, and focus on DR corresponding recommendation(s). The language of CPGs was limited to English and Chinese. If a new version of CPG had been published, the old version was excluded.
Two reviewers (YS, YT-C) independently extracted the characteristics of the included CPGs based on a standardized and pilot-tested form designed by the research group. The extracted items included the title, authors, publication year, organization, version, location of the development, funding, method of forming recommendations, the recommendations for DR screening, diagnosis, prevention, and treatment. When disagreements arose, we solved them by discussion or with the help of a third reviewer (LG).

\section{Quality assessment}

Four reviewers (YS, JC, YG, YC) evaluated the quality of the included CPGs using the Appraisal of Guidelines Research and Evaluation (second version) (AGREE II) and Reporting Items for Practice Guidelines in Healthcare (RIGHT). When addressing disagreements, we solved them by discussion.

The AGREE II instrument includes criteria to determine the methodological quality of the CPGs and consists of 23 items grouped in six domains, including scope and purpose, stakeholder involvement, rigor of development, clarity and presentation, applicability, and editorial independence [21]. The assessor must respond to 23 questions using a scale of 1 for "strongly disagree" to 7 for "strongly agree" based on examples and instructions described in the AGREE II. Each domain score was standardized as a percentage according to the following formula from the AGREE II manual. The scaled domain score $=($ obtained score - minimum possible score)/(maximum possible score - minimum possible score). Maximum possible score $=7$ (strongly agree) $\times$ number of items within a domain $\times$ number of appraisers; Minimum possible score $=1$ (strongly disagree) $\times$ number of items within a domain $\times$ number of appraisers [21].

In addition, the RIGHT checklist was used to appraise the reporting quality of CPGs. This tool consists of 22 items including basic information (items 1-4), background (items 5-9), evidence (items 10-12), recommendations (items 13-15), review and quality assurance (items 16 and 17), funding, declaration and management of interests (items 18 and 19), as well as information (items 20-22) [22]. Each item was evaluated as "Yes", "No", and "Partial" according to its own reporting content by four reviewers (YS, JC, YG, LG) independently.

\section{Data synthesis and analysis}

\section{Statistical analysis}

After completing the methodological quality evaluation by AGREE II instrument, six domain scores calculated as means. An overall evaluation of the CPG was also carried out and each CPG was classified as: "strongly 


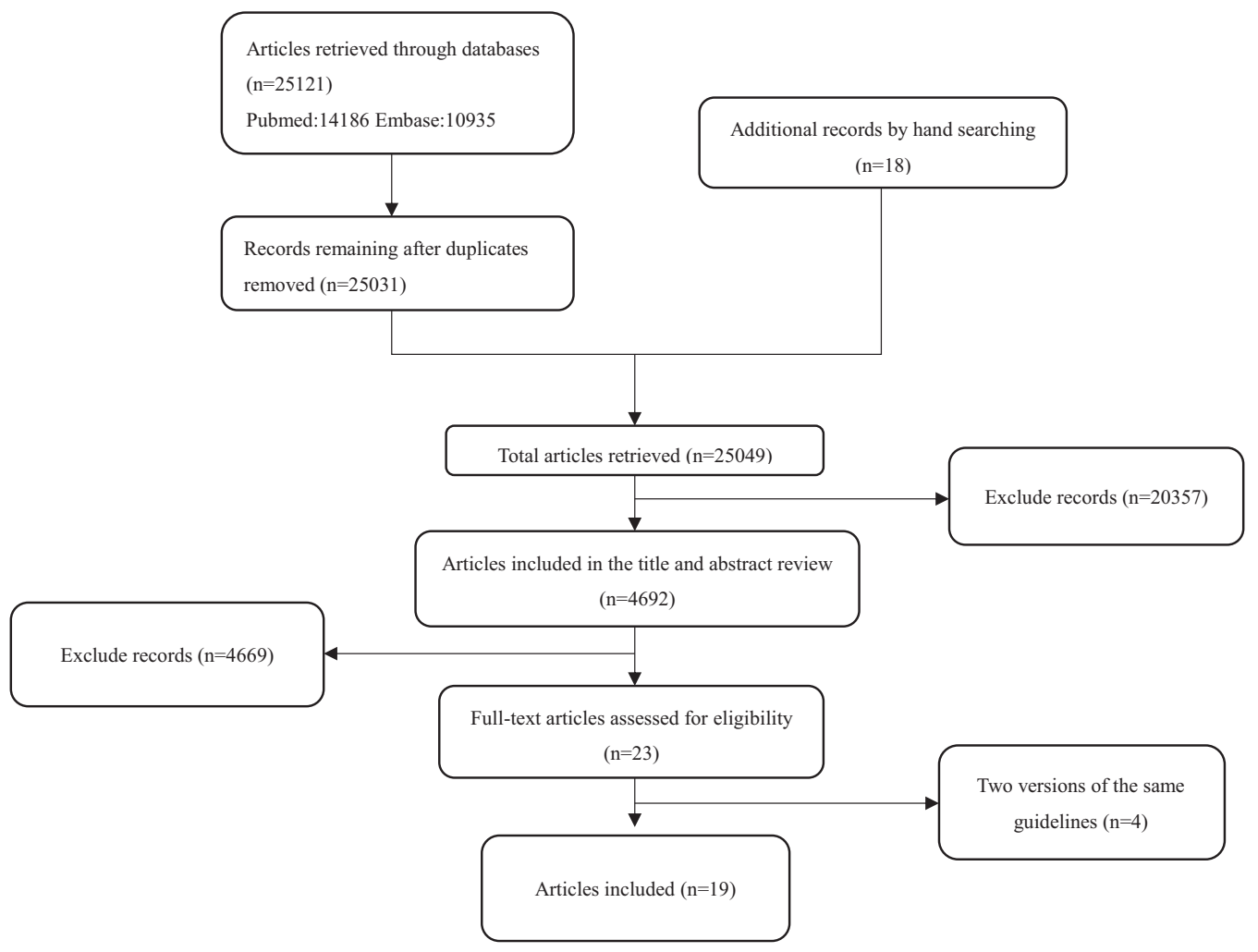

Fig. 1 Summary of evidence search and selection.

recommended" $(\mathrm{R})$ for overall scores $>60 \%$, "recommended with modifications" (RM) for scores between $30 \%$ and $60 \%$, and "not recommended" (NR) for scores $<30 \%$ [23]. Reporting quality data were presented as the number of RIGHT checklist items reported in each CPG, as well as the number of CPGs that reported individual RIGHT checklist items.

To ensure the consistency of assessment results between the four reviewers, we calculated the intraclass correlation coefficient (ICC) with the $95 \%$ confidence interval (CI) [24]. The degree of agreement between 0.01 and 0.20 was deemed minor, 0.21-0.40 fair, 0.41-0.60 moderate, $0.61-0.80$ substantial, and $0.81-1.00$ very good [20]. SPSS 22.0 software was used for statistical analysis [25].

\section{Summarizing and grading DR recommendations}

Descriptive analyses were conducted to summarize recommendations of the CPGs, including DR prevention, screening, and therapeutic strategies. To present an evidence map for explicating research trends and gaps, all analyses were conducted and bubble plots were generated by Excel 2013. With the number of the bubble proportional to the number of CPGs and the different bubble color represented the three categories of the CPGs. Green sphere for recommended CPGs, yellow sphere for recommended with modifications CPGs, and red sphere for not recommended CPGs.

\section{Results}

\section{CPGs search and characteristics}

Figure 1 shows the detailed search results of the CPGs, 19 CPGs [26-44] proved eligible. Eight CPGs were devoted to DR practices, while the rest focused on practices for diabetes mellitus and included recommendations for the DR. Of the 19 eligible CPGs, Six CPGs [28, 30, 33, 35, 36, 38] were developed by the professional diabetes society and six [26, 29, 34, 39, 41, 43] were from ophthalmological society. Two CPGs [40, 42] were developed by the professional CPG development organization. The remaining five CPGs [27, 31, 32, 37, 44] were from medical centers or health systems (Appendix 1).

\section{Analysis and grading of methodological quality and reporting quality of CPGs}

This analysis identified that the mean scores for "rigor of development" and "applicability" domains were below $50 \%$. The ICC for the assessment between the four reviewers in the study was 0.86 (95\% CI: 0.83-0.92). The highest score was $72.57 \pm 13.00$ for clarity of presentation domain (Table 1). According to the overall assessment of each CPG, 19 CPGs were divided into three levels and we use three different colored spheres to represent different 
Table 1 Standardized scores of guidelines by AGREE II instrument.

\begin{tabular}{|c|c|c|c|c|c|c|c|c|c|}
\hline No. & Organization & $\begin{array}{l}\text { Scope and } \\
\text { purpose }\end{array}$ & $\begin{array}{l}\text { Stakeholder } \\
\text { involvement }\end{array}$ & $\begin{array}{l}\text { Rigor of } \\
\text { development }\end{array}$ & $\begin{array}{l}\text { Clarity of } \\
\text { presentation }\end{array}$ & Applicability & $\begin{array}{l}\text { Editorial } \\
\text { independence }\end{array}$ & $\begin{array}{l}\text { Overal } \\
\text { Assess } \\
\text { ment }\end{array}$ & \\
\hline 1 & RCOphth [26] & 88.88 & 58.33 & 71.88 & 61.11 & 39.58 & 33.33 & 58.07 & $\mathrm{RM}$ \\
\hline 2 & ICSI [27] & 84.72 & 56.94 & 76.04 & 76.39 & 71.88 & 83.33 & 74.65 & $\mathrm{R}$ \\
\hline 3 & KDA [28] & 59.72 & 45.83 & 43.75 & 86.11 & 35.42 & 27.08 & 47.14 & RM \\
\hline 4 & AAO [29] & 80.55 & 61.11 & 71.88 & 86.11 & 52.08 & 66.67 & 67.80 & $\mathrm{R}$ \\
\hline 5 & JDA [30] & 77.77 & 58.33 & 37.50 & 48.61 & 41.66 & 54.17 & 49.65 & $\mathrm{RM}$ \\
\hline 6 & $\mathrm{MOH}$ [31] & 41.67 & 36.11 & 17.71 & 77.78 & 27.08 & 0.00 & 30.64 & RM \\
\hline 7 & MHS [32] & 76.39 & 69.44 & 50.52 & 73.61 & 31.25 & 54.17 & 54.64 & RM \\
\hline 8 & ADA [33] & 70.83 & 44.83 & 44.79 & 72.22 & 41.67 & 66.67 & 53.43 & RM \\
\hline 9 & ICO [34] & 55.56 & 36.11 & 9.38 & 69.44 & 29.17 & 0.00 & 29.78 & NR \\
\hline 10 & $\begin{array}{l}\text { ADA and EASD } \\
{[35]}\end{array}$ & 80.56 & 66.67 & 59.38 & 55.56 & 29.17 & 70.83 & 56.34 & RM \\
\hline 11 & CDA [36] & 88.89 & 86.11 & 78.13 & 83.33 & 52.08 & 91.67 & 76.30 & $\mathrm{R}$ \\
\hline 12 & ISPAD [37] & 44.44 & 22.22 & 6.25 & 63.89 & 22.92 & 50.00 & 29.86 & NR \\
\hline 13 & PDA [38] & 84.72 & 61.11 & 59.90 & 80.55 & 53.13 & 83.33 & 66.97 & $\mathrm{R}$ \\
\hline 14 & AACE [39] & 69.45 & 66.67 & 37.50 & 69.44 & 22.92 & 58.33 & 48.09 & $\mathrm{RM}$ \\
\hline 15 & NICE [40] & 100.00 & 84.72 & 88.54 & 94.29 & 83.33 & 87.50 & 88.78 & $\mathrm{R}$ \\
\hline 16 & $\operatorname{COS}[41]$ & 86.11 & 66.67 & 59.38 & 75.00 & 35.42 & 58.33 & 59.46 & RM \\
\hline 17 & SIGN [42] & 88.88 & 75.00 & 89.06 & 91.67 & 81.25 & 64.58 & 82.59 & $\mathrm{R}$ \\
\hline 18 & RANZCO [43] & 52.78 & 22.22 & 38.54 & 61.11 & 16.67 & 50.00 & 37.07 & $\mathrm{RM}$ \\
\hline \multirow[t]{2}{*}{19} & CMA [44] & 44.44 & 38.89 & 9.38 & 52.78 & 22.92 & 0.00 & 25.09 & NR \\
\hline & Mean \pm SD & $\begin{array}{l}72.44 \pm \\
17.63\end{array}$ & $55.65 \pm 18.66$ & $49.97 \pm 25.63$ & $72.58 \pm 12.66$ & $41.56 \pm 19.14$ & $52.63 \pm 28.20$ & & \\
\hline
\end{tabular}

$R$ recommended, $R M$ recommended with modifications, $N R$ not recommended.

levels. Six CPGs [27, 29, 36, 38, 40, 42] developed by ICSI, AAO, CDA, PDA, NICE, and SIGN scored higher and were classified as recommended for clinical practice, which represented by six red spheres. Ten CPGs [26, 3033, 35, 39, 41, 43] from RCOphth, KDA, JDA, MOH, MHS, ADA, ADA and EASD, AACE, COS, and RANZCO were recommended with modifications and shown by nine green spheres, and three CPGs [34, 37, 44] developed by CMA, ICO, and ISPAD were not recommended and shown by yellow spheres (Fig. 2).

The RIGHT checklist contains 22 requirements organized into seven sections with a total of 35 items. NICE and SIGN CPGs attained the largest number of reported items using RIGHT checklist, followed by CDA (32 items). In seven domains of RIGHT checklist, field one (basic information) got the highest reporting rate $(86.31 \%)$ and field five (review and quality assurance) obtained the lowest reporting rate $(40.91 \%)$ (Fig. 2).

\section{Summarizing and grading recommendations for DR}

In the bubble chart, the $Y$-axis represented the strength of the recommendations, which were divided into strong, weak and not mentioned. Different recommendations of the CPGs were represented by $X$-axis. We summarized the DR recommendations in the $\mathrm{CPG}$, including prevention, screening, pharmacotherapy, and surgical treatment.

\section{CPGs recommendations on DR prevention}

As we can see from Fig. 3, nine CPGs [26, 28$32,36,39,42]$ contained four major recommendations on DR prevention as follow:

(1) Maintaining near-normal glucose levels and nearnormal blood pressure lowers the risk of DR developing and/or progressing (Strong [29, 30, 36, 39, 42], Not mentioned [28, 31]);

(2) Blood-glucose control (HbA1c preferably $6.5-7.5 \%$ or $47.5-58.5 \mathrm{mmol} / \mathrm{mol}$ ) should be instituted to reduce the risk and progression of DR (Strong [26, 32, 42], Not mentioned [39]);

(3) Good control of blood pressure at or below 130/ $80 \mathrm{mmHg}$ should be instituted to reduce the risk and progression of DR (Strong [26, 42], Not mentioned [32]);

(4) Serum lipids control should be implemented to slow the progression of DR (Strong [26, 32, 39]). 
Fig. 2 Grading and analysis of reporting and methodological quality of CPGs.

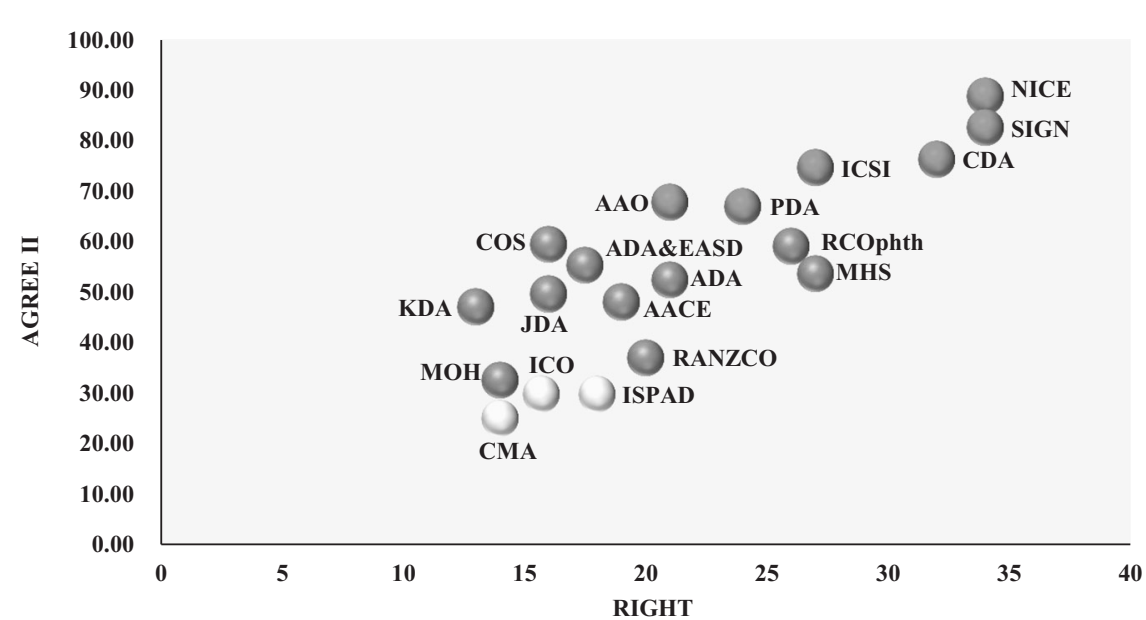

Fig. 3 Evidence map of CPG recommendations on DR prevention.

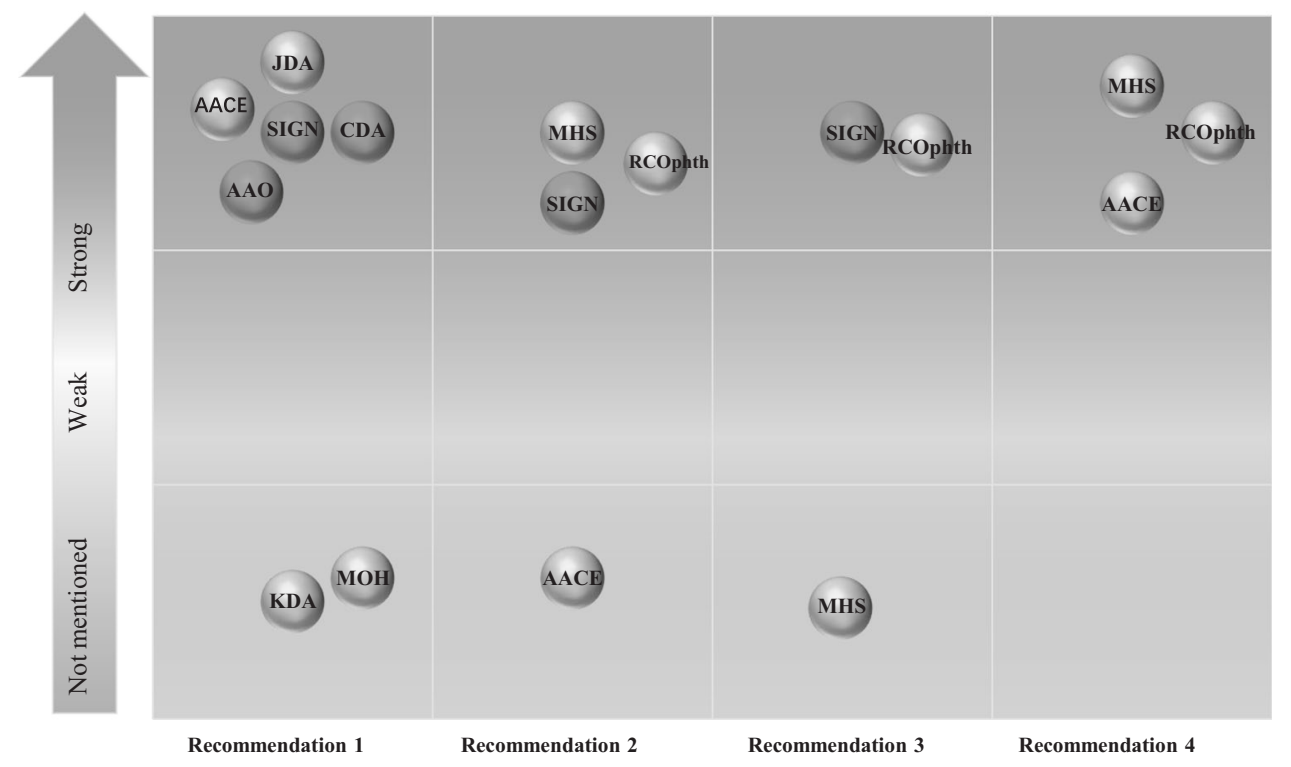

\section{CPGs recommendations on DR screening}

According to Fig. 4, nine CPGs $[26,27,29,31-$ $33,38,39,44]$ contained eight major recommendations. The summary of recommendations is as follows:

(1) Patients with diabetes should have comprehensive eye examinations periodically after the initial eye examination. If there is no evidence of DR in examinations, it is recommended to conduct the examinations every 2 years (Not mentioned [27]);

(2) People with type 1 diabetes should have annual screenings for DR beginning 5 years after the onset of their disease (Not mentioned [29, 31, 38, 39]);

(3) Patients with type 1 diabetes should have an eye examination 3-5 years after diagnosis of diabetes, and at least once yearly subsequently (Not mentioned [32]);

(4) Those with type 2 diabetes should have a prompt examination at the time of diagnosis and at least yearly examinations thereafter (Weak [32], Not mentioned $[29,31,38,39])$;

(5) For patients with well-controlled metabolic type 2 diabetes, if no retinal changes, the eye fundus may be assessed every 3 years (Not mentioned [38]);

(6) Follow-up with eyecare specialists should typically occur on an annual basis, but patients with Type 2 diabetes who have had a negative ophthalmologic examination may be screened every 2 years (Not mentioned [39]);

(7) Patients with previous type 1 or type 2 diabetes should undergo eye examinations before or during the first trimester of pregnancy, and then these patients should be monitored every trimester and for 1 year postpartum (Not mentioned [27, 32, 33]);

(8) Women who develop gestational diabetes do not require an eye examination during pregnancy and do not appear to be at increased risk of developing DR during pregnancy. However, patients with diabetes who become 


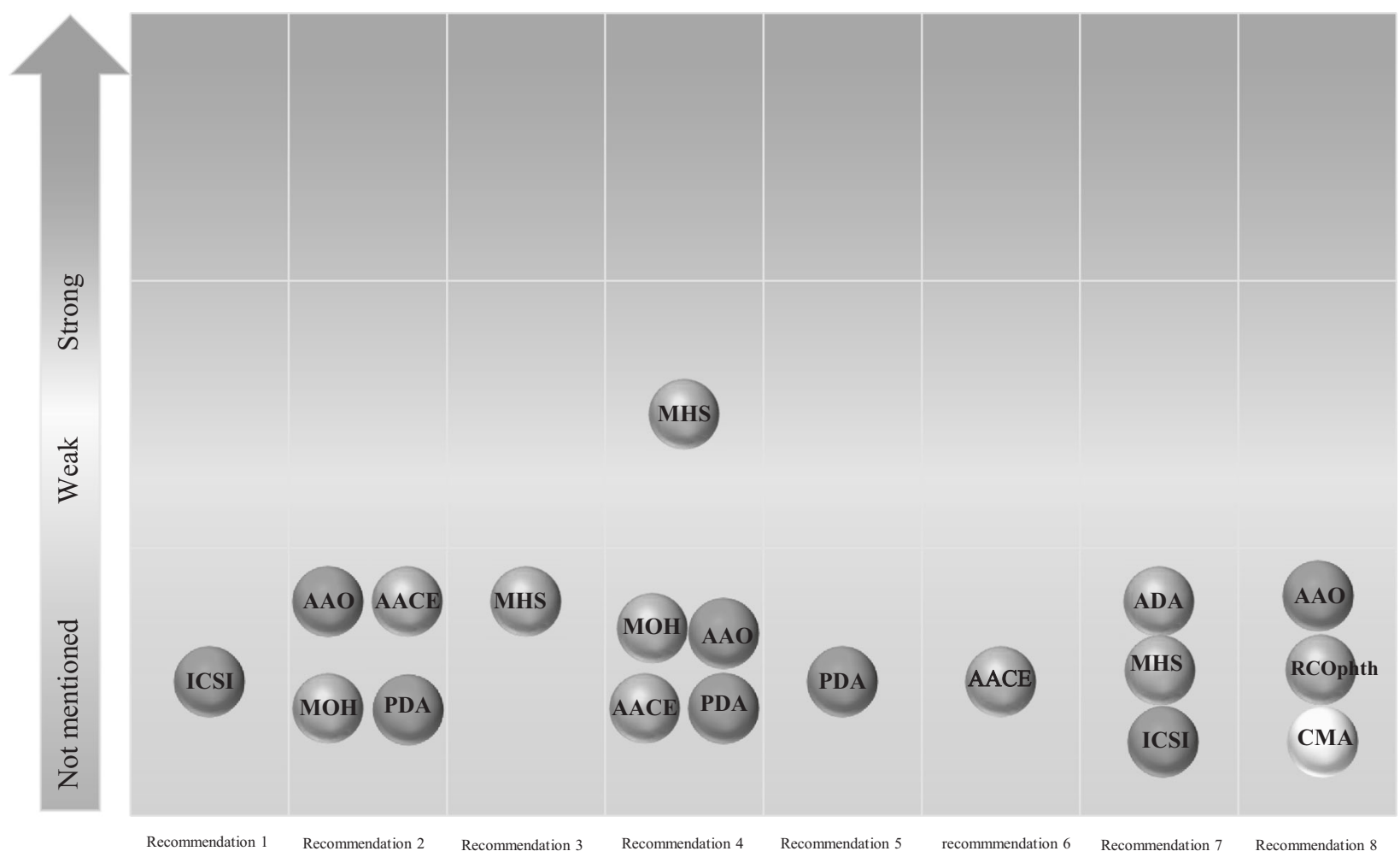

Fig. 4 Evidence map of CPG recommendations on DR screening.

pregnant should be examined early in the course of the pregnancy (Not mentioned [26, 29, 44]).

\section{CPGs recommendations on DR pharmacotherapy}

Eleven CPGs [26, 28-31, 33, 36, 38, 41, 42, 44] focused on nine recommendations (Fig. 5). The summary of recommendations is as follows:

(1) Clinical use of intravitreal triamcinolone, dexamethasone, bevacizumab, pegaptanib, and ranibizumab have been shown to be promising (Not mentioned [26]);

(2) Intravitreal injection of antivascular endothelial growth factor (anti-VEGF) preparation in the treatment of diabetic macular oedema (DME) (Strong [29], Not mentioned [28, 31, 33, 38, 42]).

(3) Intravitreal anti-VEGF therapy is sometimes recommended for patients with mild non-proliferative diabetic retinopathy (NPDR) and clinically significant macular oedema (CSME), not recommended for patients with severe NPDR and patients without DME, or patients with non-highrisk PDR (proliferative diabetic retinopathy) (Strong [29]);

(4) Intravitreal or periocular injections of steroids exerting an antiangiogenic and antioedematous effect (e.g., triamcinolone, long-acting dexamethasone, or extendedrelease fluocinolone acetonide) (Not mentioned [38]);

(5) Patients with active DR should be treated with VEGF (Not mentioned [41]);
(6) Fenofibrate slow the progression of DR and retard DR (Strong [30], Not mentioned [31, 36, 44]);

(7) The presence of DR is not a contraindication to aspirin therapy for cardio protection, as aspirin does not increase the risk of retinal hemorrhage (Not mentioned $[29,33,44])$;

(8) Acetylsalicylic acid used for cardio protection is not contraindicated in patients with DR and does not pose a risk of retinal hemorrhage (Not mentioned [38]);

(9) Calcium dobesilate improves early DR, the effect of moderate to severe DR waits for further confirmation. Some traditional Chinese medicines (compound danshen dropping pills, ginkgo tablet, fufang xueshuantong capsule and qi ming granule) have an adjuvant effect on DR treatment (Not mentioned [44]).

\section{CPGs recommendations on DR surgical treatment}

Figure 6 showed us seven recommendations on surgical treatment included in 11 CPGs [26, 28, 29, 31, 33, $34,38,39,41,42,44]$. The summary of recommendations is as follows:

(1) Laser photocoagulation is the standard of care for diabetic maculopathy and proliferative retinopathy (Strong [31], Not mentioned [26, 28, 29, 34, 38, 39]);

(2) Follow closely for development of PDR and consider early pan-retinal photocoagulation for patients at high risk 


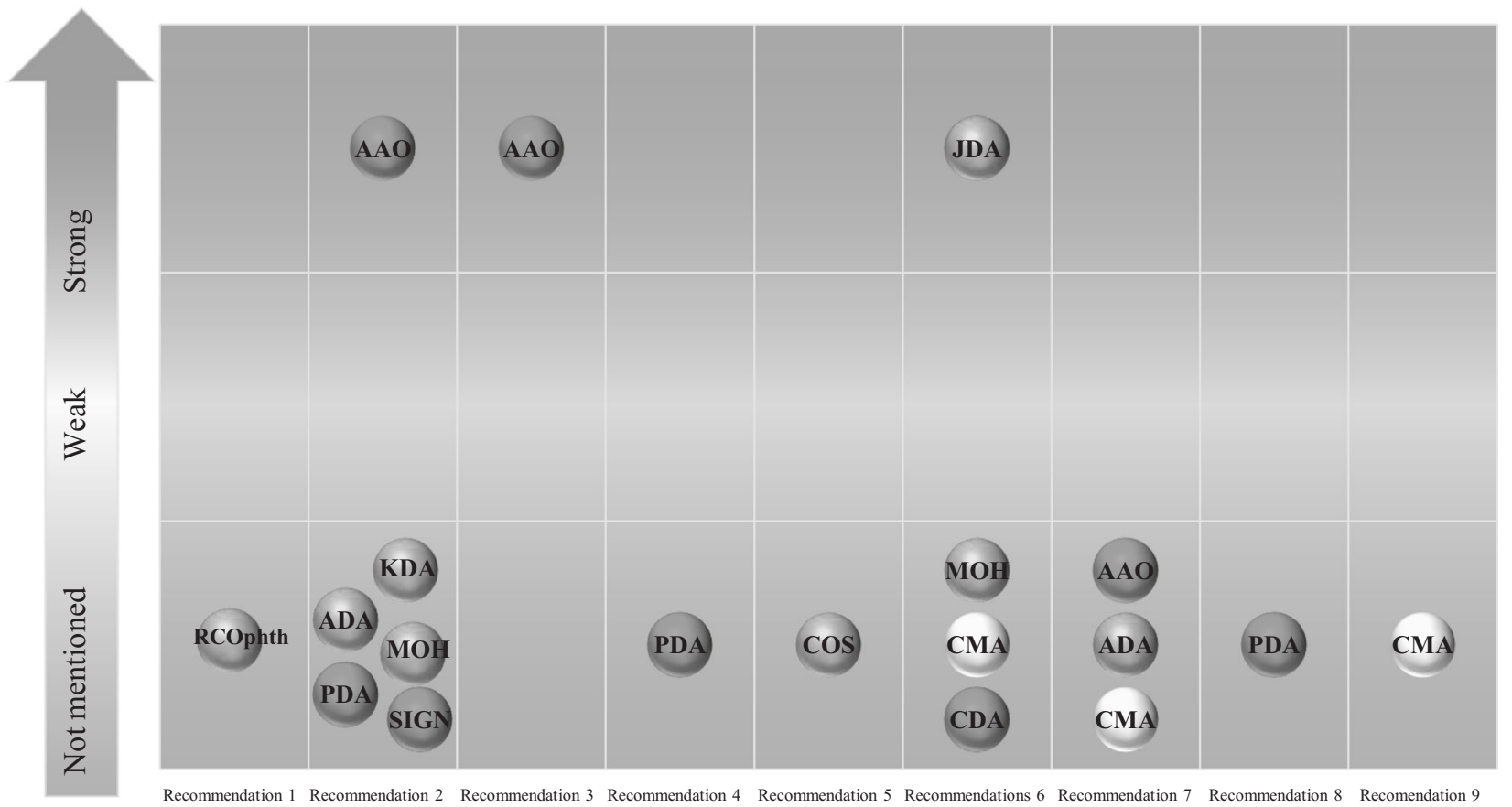

Fig. 5 Evidence map of CPG recommendations on DR pharmacotherapy.

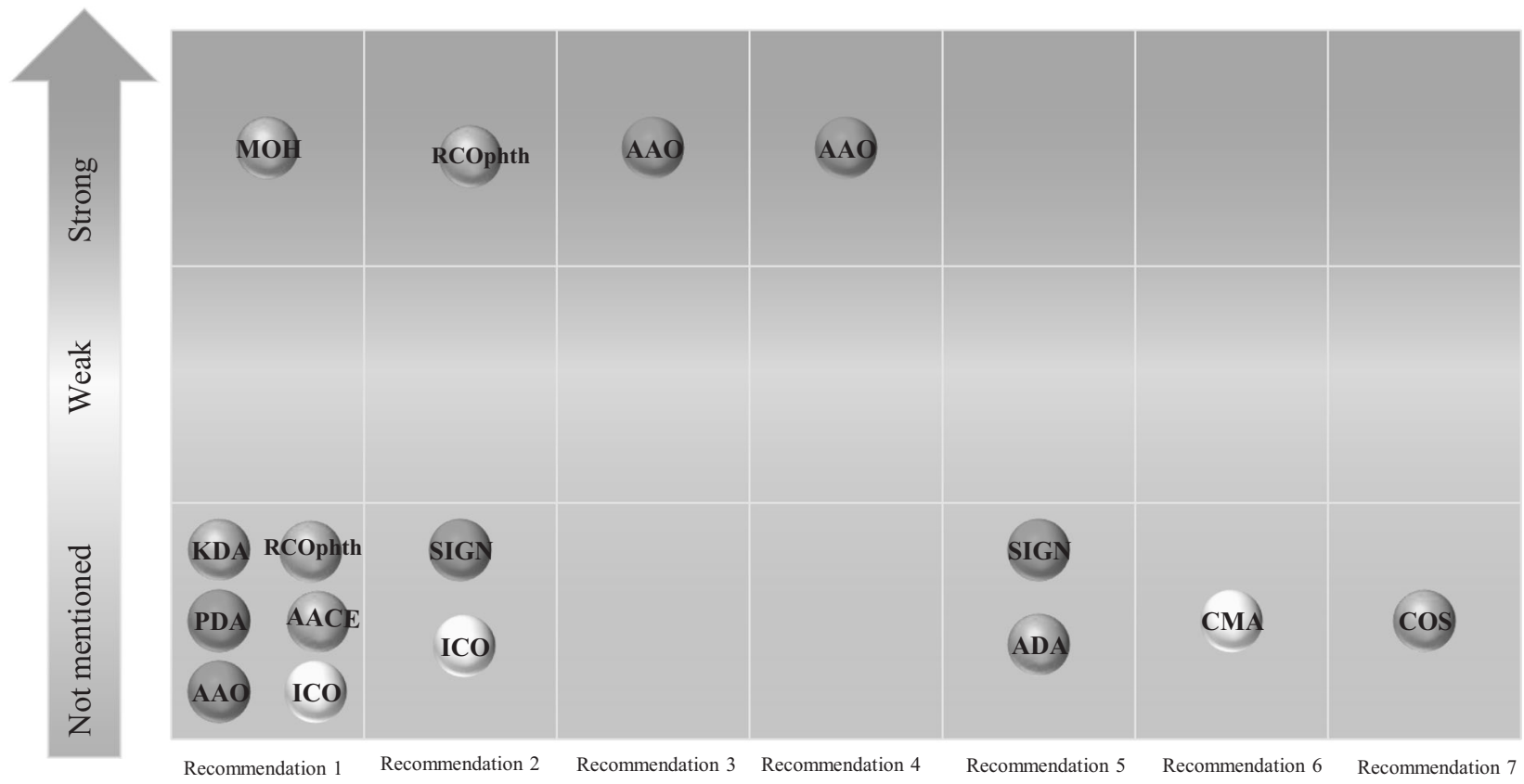

Fig. 6 Evidence map of CPG recommendations on DR surgical treatment.

of progression to PDR or poor compliance with follow-up (Strong [26], Not mentioned [34, 42]);

(3) Pan-retinal photocoagulation (PRP) is not recommended for patients with normal or minimal NPDR, mild NPDR and no DME, mild NPDR and ME, moderate NPDR and ME, moderate NPDR and no DME, mild NPDR and CSME (Strong [29]);
(4) Focal and/or grid laser treatment not recommended for patients with normal or minimal NPDR, mild NPDR and no DME, mild NPDR and ME, moderate NPDR and no DME, moderate NPDR and ME (Strong [29]);

(5) Timely laser therapy should be offered to patients with PDR and diabetic macular oedema (DME) (Not mentioned [33, 42]); 
(6) Laser photocoagulation could be used to treat severe NPDR and PDR during pregnancy (Not mentioned [44]);

(7) Intraocular injections of VEGF inhibitors are an effective treatment for DME and produce a larger gain in vision than focal or grid laser alone (Not mentioned [41]).

\section{Discussion}

This is the first systematic review of CPGs for DR using AGREE II instrument and RIGHT checklist and we used evidence map to visualize gaps. Lower quality scores were observed in the rigor of development and applicability domains, whereas all the CPGs had moderate to high scores in the purpose and objective and clarity of presentation domains. CPGs were analysed and evaluated to summarize their recommendations using standardized grades as a basis, however, we found that many CPGs did not have strict evidence and strength of recommendation or only described the level of evidence supporting the recommendations. At the same time, we found the level of evidence distribution and the strength of the recommendations varied widely between the different categories of criteria, which might confuse and hinder communication between CPGs developers [45]. CPGs were reviewed to establish the challenges likely to be encountered when managing patients with DR, including prevention, screening, pharmacotherapy, and surgical treatment. However, there were some conflicts in some organizations' CPGs.

The most commonly used grading system in CPGs for DR is the International Classification of Diabetic Retinopathy scale [25, 33, 43], which is a unified, international severity scale of disease for DR and diabetic macular oedema. Although the Early Treatment Diabetic Retinopathy Study staging system is recognized as the gold standard for staging in clinical trials in routine clinical practice, it has not been proved to be easy or practical to use [46]. The levels are characterized by the number and severity of microaneurysms, dot and blot hemorrhages, cotton wool spots (nerve fiber layer infarcts), venous abnormalities (beading and looping), and intraretinal microvascular anomalies, which are large-caliber shunt vessels within non-perfused regions of the capillary bed [47]. Imaging modalities, especially optical coherence tomography (OCT) and fluorescein angiography (FA), play a crucial role in the diagnosis and management of complications of DR at present. If available, OCT imaging may be used, but its utility as a primary screening tool is yet to be established [29]. It is likely to be more widely used over the next few years. Particularly for DME and subtle NV (neovascularization), OCT is the most sensitive method to identify DME. OCT could provide a quantitative assessment of DME to determine its severity of DME. Retinal map scan is useful in locating the retinal thickening area. Single scan is valuable in detailing the types of DME as diffuse, cystic changes, subretinal fluid/retinal detachment, and vitreoretinal traction [33]. Newer modalities of OCT, including optical coherence tomography angiography, are expected for the further expanding role of imaging. FA could be used as a guide to evaluate the retinal nonperfusion area, presence of retinal neovascularization, and microaneurysms or macular capillary non-perfusion in DME [33]. However, this classification seems to be inadequate in the clinical practice [48].

Early detection and treatment of progressive retinal disease are significant aspects of the management [49]. The aetiology of DR is multifactorial, and the main causes may be chronic capillary perfusion and retinal ischemia [50]. From a clinical point of view, it is clear that screening strategies depend on the rate of incidence and progression of DR and on risk factors altering the rate. For patients with diabetes, regular follow-up with early detection and treatment of vision-threatening DR enables the prevention of visual loss caused by DR up to $98 \%$. For patients with diabetes, regular follow-up with early detection and treatment of vision-threatening DR enables the prevention of up to $98 \%$ of visual loss due to DR [51]. Thus, screening for $\mathrm{DR}$ at the right time is urgently needed. It is recommended in most CPGs (AAO, MOH, PDA, and AACE) that patients with Type 1 diabetes should have an annual screening for DR during the first 5 years after the onset of their disease. However, MHS CPGs have suggested that Type 1 diabetic patients should have an optical examination 3-5 years after diagnosis of diabetes, and at least once yearly subsequently [32]. Those with Type 2 diabetes should have a prompt examination at the time of diagnosis and at least once yearly thereafter [29, 31, 32, 38, 39]. On the basis of CPGs by PDA, patients with type 2 diabetes under excellent metabolic control and without retinal changes, the ocular fundus may be assessed every 3 years, which will significantly reduce the costs of treatment for diabetic patients [38]. Taking the financial costs for health care systems globally into consideration, it is suggested in CPGs by ADA that reducing the examination frequency (every 2 years) may be considered, if there are adequate glucose control and normal ocular examination [33].

In practice, timing and frequency of ocular examinations in people with diabetes are often individual. Three CPGs recommend that ocular examinations should be performed before pregnancy or in the first trimester in patients with type 1 or type 2 diabetes, and then these patients should be monitored every trimester and for 1 year after delivery as indicated by the degree of retinopathy [27, 32, 33]. RCOphth, AAO, and CMA CPGs [26, 29, 44] have suggested that women with gestational diabetes do not require an ocular examination during pregnancy and there will not 
be a higher risk of DR. Recommendations on ocular examinations of DR during pregnancy are consistent among CPGs.

There are eight important risk factors for DR in CPGs. Long duration of diabetes and poor hypertension control are well-known risk factors for DR. Results of previous epidemiological evidence in CPGs have also shown that DR is associated with many other systemic and lifestyle factors, including nephropathy, obesity, alcohol consumption, and hematological markers of anemia, hyperlipidemia, and hyperglycemia. Harjutsalo et al. found that the onset age of type 1 diabetes is associated with the age of menarche, which would increase the risk of diabetic nephropathy and severe retinopathy later in life [52]. Similarly, pregnancy is associated with deterioration of DR. Therefore, patients with type 1 diabetes should consider planned dilated retinal examination after puberty and during pregnancy. These results suggest that the presence of DR is a marker of widespread microcirculation damage in end organ. In addition, there is a need to improve cardiovascular monitoring and lifestyle follow-up in patients with DR [53].

According to the recommendations in CPGs for DR, the pharmacotherapy of DR is consistent and could be summarized in nine major items. However, there is no currently approved drug treatment directly targeting DR. Clinical application of intravitreal triamcinolone, dexamethasone, bevacizumab, pegaptanib, and ranibizumab has been shown to be promising. Preparation for intravitreal injection of anti-VEGF preparation has been suggested by six CPGs in the treatment of DME [28, 29, 31, 33, 38, 42]. Although anti-VEGF therapy has a good clinical application prospect in the treatment of DR, its long-term safety in patients with diabetes has not been determined in CPGs. Local adverse events of intravitreal anti-VEGF therapy include cataract formation, retinal detachment, vitreous hemorrhage, infection, and potential loss of neural retinal cells [54]. AAO CPG have shown that intravitreal anti-VEGF therapy is recommended for patients with mild NPDR and CSME, but not recommended for patients with severe NPDR or nonhigh-risk PDR and patients without DME. Intravitreal or periocular injections of steroids (including triamcinolone, long-acting dexamethasone, or extended-release fluocinolone acetonide) have an antiangiogenic and antioedematous effect. A systematic review has found that, in the shortterm, intravitreal injection of triamcinolone acetonide (a kind of long-acting corticosteroid) is effective in improving visual acuity in patients with refractory DME, but the effects could not last long [55]. It is recommended in four CPGs (including JDA, CDA, MOH, and CMA) that fenofibrate can slow the progression of retinopathy and retard DR [30, 31, 36, 44]. AAO, ADA, CMA, and CPGs have emphasized that DR is not a contraindication to aspirin therapy for cardio protection, as aspirin cannot increase the risk of retinal hemorrhage [29, 33, 44]. For the prevention and treatment of diseases, combination of traditional Chinese and western medicine is suggested in CPGs by CMA. Qiming granule, compound Danshen dripping pills, ginkgo leaf, and compound Xueshuantong capsule have the function of adjuvant therapy, which is recommended in CPGs developed by CMA [44]. The main ingredients of these Chinese medicines are Milkvetch Root, Dan-Shen Root, Panax notoginseng, and ginkgo. Many research results have proved that these Chinese medicines could regulate blood sugar levels dual directionally. At the same time, they could also dilate arterial blood vessels and small blood vessels [56-58].

Recommendations regarding surgical therapy and timing choice in CPGs are mostly consistent, but remain unspecific on some details. Vitrectomy is a main surgical treatment for two blinding complications of advanced retinopathy, persistent vitreous hemorrhage, and retraction retinal detachment [26, 28, 29, 31, 34, 38, 39]. Meanwhile, the surgical treatment can be applied to patients with type 1 diabetes and persistent vitreous hemorrhages [41].

Since the advent of the ophthalmic laser in the 1960s, pan-retinal photocoagulation has become a main treatment method for DR [59]. Laser therapy has long been considered as an evidence-based treatment for DME and PDR. Even though new therapies such as anti-VEGF and intravitreal injections of corticosteroid are changing the outlook for DME therapy. A study showed that the prevalence of severe visual loss at 2 years was reduced by $50 \%$ in patients who received prompt pan-retinal photocoagulation compared with observation [60]. Although lasers are effective in preventing vision loss in a timely and appropriate manner, their destructiveness will cause severe side effects on eyes. As a result, researchers continue to search for new and more effective treatment strategies aimed at improving vision without damaging tissue. According to RCOphth, AACE, KDA, AAO, MHS, MOH, ICO, and PDA CPGs, laser photocoagulation is the standard for the treatment of diabetic maculopathy and proliferative DR, which can reduce the risk of vision loss in patients [26, 28, 31, 32, 34, 38, 39]. The results showed that timely and appropriate laser treatment could reduce the loss vision of these patients by $50 \%$. The laser clinic should only be staffed by appropriate ophthalmologists with required knowledge, experience, and skills of retinal laser treatments [26]. According to the AACE CPG recommendation, anretinal scatter laser photocoagulation is the treatment of choice for high-risk proliferative retinopathy [39]. For macular oedema, the combination of focal laser photocoagulation with intravitreal antivascular endothelial growth factor modalities appear to offer optimal benefit. At the same time, AAO CPG clearly presented that, according to the degree of classification, pan-retinal photocoagulation is not 
recommended for patients with normal or minimal NPDR, mild NPDR and no DME, mild NPDR and ME, moderate NPDR and ME, moderate NPDR and no DME, mild NPDR and CSME [29]. Focal and/or grid laser treatment is not recommended for patients with normal or minimal NPDR, mild NPDR and no DME, mild NPDR and ME, moderate NPDR and no DME, moderate NPDR and ME. Different CPGs on laser therapy recommendations to patients with proliferative DR and diabetic macular oedema vary in detail. However, the presentation of recommendations was relatively clear and specific, and applicable to clinicians. KDA CPG has clearly described management recommendations for patients with DR, which provides guidance for a preferred practice pattern for the general population of patients with diabetes [28]. The population was further distinguished and lists laser parameters. For example, it has proposed that if DME is associated with large areas of macular ischemia, only the areas of retinal thickening should be treated. The expression form selected by the laser photocoagulation is very user-friendly for the clinicians [33]. We should follow closely for development of PDR and consider early pan-retinal photocoagulation for patients at high risk of progression to PDR or with poor compliance with follow-up [34, 42]. Severe visual impairment (legal blindness) could be reduced through laser photocoagulation for people with severe or very severe non-proliferative DR, which is also effective in new vessels elsewhere with vitreous hemorrhage and new vessels elsewhere without vitreous hemorrhage in people with type 2 diabetes [33, 42]. Female patients with diabetes, or in pregnancy will accelerate the occurrence and development of DR. Laser photocoagulation could be used to treat severe NPDR and PDR during pregnancy [60]. CMA CPG has suggested that laser photocoagulation could be used to treat severe NPDR and PDR during pregnancy, and whether to choose laser treatment or not depends on the severity of DR and incorporate into DME or not [44]. These recommendations from CPGs by CMA are not clear and specific. Recommendations should be clearly described in detail, including the application circumstances, and suitable patients, and should be supported by evidence [21].

The results of this evidence mapping could be used to identify, collect and evaluate the characteristics of published CPGs and promote improvement in the development of trustworthy CPGs for DR. With some recommendations inconsistent, at the same time, we found that some CPGs might be silent on certain matters, which might confuse CPGs users. In view of this situation, we have conducted indepth thinking and exploration. First, we found that the level of distribution of evidence and the strength of recommendations in CPGs varied widely between different categories of criteria (Appendix 2). In DR CPG, different grading systems used to classify the quality and strength of the evidence were recommended. For example, some recommendation systems only focus on the quality of evidence, however, the strength of recommendations in GRADE (The Grading of Recommendations Assessment, Development and Evaluation) system reflects the extent to which we could be confident that the composite desirable effects of a management strategy outweigh the composite undesirable effects. The difference between systems in CPGs may lead to different final recommendations. Secondly, policies and patient's preference in different countries remain inconsistent, which also inverts that the application of evidence-based evidence should be localized, and the promotion content of the CPGs will be different [61]. For example, the Chinese government encourages the development of the discipline of traditional Chinese medicine, and researches have also found that traditional Chinese medicine play a positive role in the treatment of diseases [62]. Therefore, there are recommendations for traditional Chinese medicine in the CPGs developed by China. Through the development of standardized systematic reviews, collection of evidence resources, and finally CPGs were development, but differences in the source of evidence at the beginning may lead to final clinical decisions. Finally, one thing that we may overlook is evidence source. These recommendations in CPGs were clearly related to the supporting evidence. It is found that even for the same recommendation, the supporting evidences are not exactly the same, and the database of evidence retrieval in each CPG is absolutely inconsistent. For example, during the development of the JDA evidence, in addition to searching Medline databases, local Japanese ICHUSHI database is also included [30]. Therefore, a standardized grading system, such as GRADE should be used on the level of evidence and the strength of recommendation to provide clear information. Methodologists from the development team of CPGs should pay attention to the source of evidence and comprehensive in the process of evidence collection in the database. During the promotion of the CPGs, we must pay attention to the evidence application mechanism and localization promotion strategies in different country scenarios.

The results of this evidence mapping could be used to identify, collect, and evaluate the characteristics of published CPGs and then promote improvement in the development of trustworthy CPGs for DR.

Acknowledgements The authors appreciate Xiaoqing Wang and Nan Yang (Chinese GRADE Center) for their methodological support and assistance with the final editing of the article.

Author contributions Y.S., and J.H.-T., did the study design. Y.T.-C., and J.H.-T. performed literature search. Y.S and J.C. contributed to the data acquisition. Y.S., and J.C. contributed to data interpretation and statistical analysis. L.G., and J.H.-Z., supervised and provide mentorship. Y.S., and Y.T.-C. wrote the first draft of the report. Y.S., and 
J.B.-X., edited the report and all authors contributed to revision of the report. All authors reviewed the paper, approved the final draft, and agreed to submit it for publication.

\section{Compliance with ethical standards}

Conflict of interest The authors declare that they have no conflict of interest.

Publisher's note Springer Nature remains neutral with regard to jurisdictional claims in published maps and institutional affiliations.

\section{References}

1. Shaw JE, Sicree RA, Zimmet PZ. Global estimates of the prevalence of diabetes for 2010 and 2030. Diabetes Res Clin Pract. 2010;87:4-14.

2. Cheung N, Mitchell P, Wong TY, Wong TY. Diabetic retinopathy. Lancet. 2010;376:124-34.

3. Whiting DR, Guariguata L, Weil C, Shaw J. IDF diabetes atlas: global estimates of the prevalence of diabetes for 2011 and 2030. Diabetes Res Clin Pract. 2011;94:311-21.

4. Do DV, Wang X, Vedula SS, Marrone M, Sleilati G, Hawkins BS, et al. Blood pressure control for diabetic retinopathy. Cochrane Database Syst Rev. 2015;1:CD006127.

5. Zhang HW, Zhang H, Grant SJ, Wan X, Li G. Single herbal medicine for diabetic retinopathy. Cochrane Database Syst Rev. 2018;12:CD007939.

6. Frank RN. Diabetic retinopathy. N Eng J Med. 2004;350:48-58.

7. Kahn HA, Hiller R. Blindness caused by diabetic retinopathy. Am J Ophthalmol. 1974;78:58-67.

8. Stolk RP, Vingerling JR, De Jong PTVM, Dielemans I, Hofman A, Lamberts SW, et al. Retinopathy, glucose, and insulin in an elderly population: the rotterdam study. Diabetes. 1995;44:11-5.

9. Song P, Yu J, Chan KY, Theodoratou E, Rudan I. Prevalence, risk factors and burden of diabetic retinopathy in China: a systematic review and meta-analysis. J Glob Health. 2018;8:010803.

10. Saaddine JB, Honeycutt AA, Narayan KM, Zhang X, Klein R, Boyle JP. Projection of diabetic retinopathy and other major eye diseases among people with diabetes mellitus: United States, 2005-2050. Arch Ophthalmol. 2008;126:1740-7.

11. Owolabi MO, Yaria JO, Daivadanam M, Makanjuola AI, Parker G, Oldenburg B, et al. Gaps in guidelines for the management of diabetes in low- and middle-income versus high-income countries —a systematic review. Diabetes Care. 2018;41:1097-105.

12. Grimshaw J, Freemantle N, Wallace S, Russell I, Hurwitz B, Watt I, et al. Developing and implementing clinical practice guidelines. Qual Saf Health Care. 1995;4:55-64.

13. Bennett WL, Odelola OA, Wilson LM, Bolen S, Selvaraj S, Robinson KA, et al. Evaluation of guideline recommendations on oral medications for type 2 diabetes mellitus. Ann Intern Med. 2012;156:27.

14. Hakkennes S, Dodd K. Guideline implementation in allied health professions: a systematic review of the literature. Qual Saf Health Care. 2008;17:296-300.

15. Petroni A, Corso A, Melano R, Rossi A, Galas M. Effectiveness and efficiency of guideline dissemination and implementation strategies. Health Technol Assess. 2004;8:1-72.

16. Ge L, Tian JH, Li YN, Pan JX, Li G, Wei D, et al. Association between prospective registration and overall reporting and methodological quality of systematic reviews: a meta-epidemiological study. J Clin Epidemiol. 2018;93:45-55.
17. Erickson J, Sadeghirad B, Lytvyn L, Slavin J, Johnston BC. The scientific basis of guideline recommendations on sugar intake: a systematic review. Ann Intern Med. 2017;166:257-67.

18. Lam J, Howard BE, Thayer K, Shah RR. Low-calorie sweeteners and health outcomes: a demonstration of rapid evidence mapping (rEM). Environ Int. 2019;123:454-8.

19. Hetrick SE, Parker AG, Callahan P, Purcell R. Evidence mapping: illustrating an emerging methodology to improve evidence-based practice in youth mental health. J Eval Clin Pract. 2010;16:1025-30.

20. Graham R, Miller Wolman D, Greenfield S, et al. Clinical practice guidelines we can trust. Institute of Medicine (US) Committee on Standards for developing trustworthy clinical practice guidelines. Washington (DC): The National Academies press (US); 2011.

21. AGREE Next Steps Consortium. The AGREE II Instrument [2019-04-02]. 2011. http://www.agreetrust.org.

22. Chen Y, Yang K, Marusic A, Qaseem A, Meerpohl J, Flottorp S, et al. A reporting tool for practice guidelines in health care: the RIGHT statement. Ann Intern Med. 2017;166:128-32.

23. Jiang M, Guan WJ, Fang ZF, Xie YQ, Xie JX, Chen H, et al. A critical review of the quality of cough clinical practice guidelines. Chest. 2016;150:777-88.

24. Bartko JJ. The intraclass correlation coefficient as a measure of reliability. Psychol Rep. 1966;19:3-11.

25. Alonso-Coello P, Irfan A, Sola I, Gich I, Delgado-Noguera M, Rigau D, et al. The quality of clinical practice guidelines over the last two decades: a systematic review of guideline appraisal studies. Qual Saf Health Care. 2010;19:e58.

26. The Royal College of Ophthalmologists. Diabetic retinopathy guidelines. 2012.

27. Institute for Clinical Systems Improvement (ICSI). Diagnosis and management of type 2 diabetes mellitus in adults. 2014

28. Korean Diabetes Association (KDA). Treatment guidelines for diabetes. 2015.

29. American Academy of Ophthalmology. Diabetic retinopathy. 2014.

30. Haneda M, Noda M, Origasa H, Noto H, Yabe D, Fujita Y, et al. Japanese clinical practice guideline for diabetes 2016. Diabetol Int. 2018;9:1-45.

31. Ministry of Healthy. Diabetic retinal screening, grading, monitoring and referral guidance. 2016.

32. Goh SY, Ang SB, Bee YM, Chen YT, Gardner DS, Ho ET, et al. Ministry of health clinical practice guidelines: diabetes mellitus. Singap Med J. 2014;55:334-47.

33. American Diabetes Association (ADA). Standards of medical care in diabetes. 2017.

34. International Council of Ophthalmology. ICO guidelines for diabetic eye care. 2017.

35. American Diabetes Association (ADA) and the European Association for the Study of Diabetes (EASD). Management of hyperglycemia in type 2 diabetes. 2018.

36. Korean Diabetes Association (KDA). Treatment guidelines for diabetes. 2015

37. Mahmud FH, Elbarbary NS, Fröhlich-Reiterer E, Holld ReinhardW, Kordonourie Olga, Knipf Mikael, et al. Other complications and associated conditions in children and adolescents with type 1 diabetes. Pediatr Diabetes. 2018;19:275-86.

38. Polish Diabetes Association (PDA). 2018 Guidelines on the management of diabetic patients. A position of Diabetes Poland. 2018.

39. Handelsman Y, Bloomgarden ZT, Grunberger G, Umpierrez G, Zimmerman RS, Bailey TS, et al. American association of clinical endocrinologists and american college of endocrinology-clinical practice guidelines for developing a diabetes mellitus comprehensive care plan-2015. Endocr Pract. 2015;21 Suppl 1:1-87. 
40. Diabetes (type 1 and type 2) in children and young people: diagnosis and management. Diabetic foot problems: prevention and management. London (UK); 2015.

41. Hooper P, Boucher MC, Cruess A, Dawson KG, Delpero W, Greve M, et al. Excerpt from the Canadian Ophthalmological Society evidence-based clinical practice guidelines for the management of diabetic retinopathy. Can J Ophthalmol. 2017;52 Suppl 1:S45-74.

42. Scottish Intercollegiate Guidelines Network (SIGN). Management of diabetes A national clinical guideline. Part of NHS quality improvement Scotland. 2010.

43. Royal Australian and New Zealand College of Ophthalmologists. Diabetic macular oedema: evidence-based treatment recommendations for Asian countries. 2017.

44. Chinese Medical Association. Expert consensus on the prevention and treatment of diabetic retinopathy. 2018.

45. Shen WQ, Yao L, Wang XQ, Hu Y, Bian ZX. Quality assessment of cancer cachexia clinical practice guidelines. Cancer Treat Rev. 2018;70:9-15.

46. International Clinical Classification System for Diabetic Retinopathy and Diabetic Macular Edema-2012.

47. Stitt AW, Lois N, Medina RJ, Adamson P, Curtis TM. Advances in our understanding of diabetic retinopathy. Clin Sci. 2013;125:1-17.

48. Bandello F, Battaglia PM, Tremolada G, Lattanzio R, De Benedetto U, Iacono P. Steroids as part of combination treatment: the future for the management of macular edema? Ophthalmologica. 2010;224 Suppl 1:41-5.

49. Park YG, Roh YJ. New diagnostic and therapeutic approaches for preventing the progression of diabetic retinopathy. J Diabetes Res. 2016;2016:1753584.

50. Powers M, Greven M, Kleinman R, Nguyen QD, Do D. Recent advances in the management and understanding of diabetic retinopathy. F1000Res. 2017;6:2063.

51. Ferris FL 3rd. How effective are treatments for diabetic retinopathy? JAMA. 1993;269:1290-1.
52. Harjutsalo V, Maric-Bilkan C, Forsblom C, Groop PH. Age at menarche and the risk of diabetic microvascular complications in patients with type 1 diabetes. Diabetologia. 2016;59:472-80.

53. Cheung N, Mitchell P, Wong TY. Diabetic retinopathy. Lancet. 2010;376:124-36.

54. Lally DR, Shah CP, Heier JS. Vascular endothelial growth factor and diabetic macular edema. Surv Ophthalmol. 2016;61: 759-68.

55. Yilmaz T, Weaver CD, Gallagher MJ, Corderocoma M, Cervantescastaneda RA, Klisovic D, et al. Intravitreal triamcinolone acetonide injection for treatment of refractory diabetic macular edema: a systematic review. Ophthalmology. 2009;116:902-11.

56. Zhao JX, Wang SD, Pang B, Tan Q. Standards for diagnosis and treatment of traditional Chinese medicine for diabetes with hypertension. World Journal of Integrated Traditional and Western Medicine. 2011;6:638-44.

57. Deng H, Jing M, Yuan W, Zhan MX, Jing PW. Clinical observation on treating early diabetic retinopathy with compound danshen dripping pills. Chin J Ophthalmol. 2005;15:72-5.

58. Xing YW, Zou JJ, Shi YQ, Liu ZM. Protection of Fufang Xueshuantong capsule against retinal oxidative damage of diabetic rats. J Med Res. 2016;45:40-3.

59. Heng LZ, Comyn O, Peto T, Tadros C, Ng E, Sivaprasad S, et al. Diabetic retinopathy: pathogenesis, clinical grading, management and future developments. Diabet Med. 2013;30:640-50.

60. Photocoagulation treatment of proliferative diabetic retinopathy. Clinical application of Diabetic Retinopathy Study (DRS) findings, DRS Report Number 8. The Diabetic Retinopathy Study Research Group. Ophthalmology. 1981; 88:583-600.

61. Sun Y, Gao Y, Chen J, Sun H, Cai YT, Ge L, et al. Evidence mapping of recommendations on diagnosis and therapeutic strategies for diabetes foot: an international review of 22 guidelines. Metabolism. 2019;100:153956.

62. Feng ZP, Tao QS, Peng DY. Evolution characteristics and enlightenment of self-innovation of traditional Chinese medicine industry. Chin J Chin Materia Medica. 2015;40:2252-7. 